\title{
INEXACT AND PRECONDITIONED UZAWA ALGORITHMS FOR SADDLE POINT PROBLEMS*
}

\author{
HOWARD C. ELMAN ${ }^{\dagger}$ AND GENE H. GOLUB ${ }^{\ddagger}$ \\ Dedicated to Seymour Parter on the occasion of his 65th birthday.
}

\begin{abstract}
Variants of the Uzawa algorithm for solving symmetric indefinite linear systems are developed and analyzed. Each step of this algorithm requires the solution of a symmetric positivedefinite system of linear equations. It is shown that if this computation is replaced by an approximate solution produced by an arbitrary iterative method, then with relatively modest requirements on the accuracy of the approximate solution, the resulting inexact Uzawa algorithm is convergent, with a convergence rate close to that of the exact algorithm. In addition, it is shown that preconditioning can be used to improve performance. The analysis is illustrated and supplemented using several examples derived from mixed finite element discretization of the Stokes equations.
\end{abstract}

Key words. saddle point, Uzawa, inexact, inner/outer, preconditioned, Stokes

AMS subject classifications. primary 65F10, 65N20; secondary 15A06

1. Introduction. Consider the linear system of equations

$$
\left(\begin{array}{cc}
A & B^{T} \\
B & -C
\end{array}\right)\left(\begin{array}{l}
u \\
p
\end{array}\right)=\left(\begin{array}{l}
f \\
0
\end{array}\right)
$$

where $A$ is symmetric positive definite and $C$ is symmetric positive semidefinite. Problems in this class arise frequently in the context of minimization of quadratic forms subject to linear constraints; see, e.g., [13], [18], and [35]. In this paper, we develop and analyze variants of the classical Uzawa algorithm for solving (1).

We briefly review this method here; see, e.g., [2], [13], and [16] for more details, and [21] for a recent treatment for use with spectral methods. Starting with an initial approximation $p_{0}$ of $p$, the Uzawa algorithm constructs a sequence of approximations $u_{k} \approx u$ and $p_{k} \approx p$ as follows:

$$
\begin{aligned}
& \text { for } k=0 \text { until convergence, do } \\
& \quad \text { Solve } A u_{k+1}=f-B^{T} p_{k} \\
& \quad \text { Compute } p_{k+1}=p_{k}+\alpha\left(B u_{k+1}-C p_{k}\right) \\
& \text { enddo }
\end{aligned}
$$

Elimination of $u_{k+1}$ from the construction of $p_{k+1}$ gives the iteration

$$
p_{k+1}=p_{k}+\alpha\left(B A^{-1} f-\left(B A^{-1} B^{T}+C\right) p_{k}\right)
$$

for the unknowns $\left\{p_{k}\right\}$. This is a fixed-parameter first-order Richardson iteration [28, p. 141] applied to the system

$$
\left(B A^{-1} B^{T}+C\right) p=B A^{-1} f .
$$

* Received by the editors May 28, 1993; accepted for publication December 1, 1993. This work was supported by U. S. Army Research Office grant DAAL-0392-G-0016 and National Science Foundation grant ASC-8958544 at the University of Maryland, National Science Foundation grant CCR-8821078 at Stanford University, and Science and Engineering Research Council of Great Britain Visiting Fellowship grant GR/J 04982 at the University of Manchester Institute of Science and Technology.

$\dagger$ Department of Computer Science and Institute for Advanced Computer Studies, University of Maryland, College Park, Maryland 20742 (elman@cs.umd.edu).

$\ddagger$ Department of Computer Science, Stanford University, Stanford, California 94305 (golub@sccm.stanford.edu). 
Good choices of the scalar $\alpha$ are determined from this observation. Equations (3)-(4) imply that the errors satisfy

$$
p-p_{k}=\left(I-\alpha\left(B A^{-1} B^{T}+C\right)\right)^{k}\left(p-p_{0}\right) .
$$

Let $(x, y)$ denote the Euclidean vector inner product, $\|x\|_{2}$ the associated norm, and $\|X\|_{2}$ the induced matrix norm. Since $B A^{-1} B^{T}+C$ is symmetric, it follows that $\rho\left(I-\alpha\left(B A^{-1} B^{T}+C\right)\right)=\left\|I-\alpha\left(B A^{-1} B^{T}+C\right)\right\|_{2}$, so that the error norm satisfies

$$
\left\|p-p_{k}\right\|_{2} \leq\left[\rho\left(I-\alpha\left(B A^{-1} B^{T}+C\right)\right)\right]^{k}\left\|p-p_{0}\right\|_{2} .
$$

Let $\lambda_{1}$ denote the minimum eigenvalue of $B A^{-1} B^{T}+C$, and $\lambda_{2}$ its maximum eigenvalue. The Uzawa algorithm is convergent provided $\rho\left(I-\alpha\left(B A^{-1} B^{T}+C\right)\right)<1$, i.e., $0<\alpha<2 / \lambda_{2}$. We will take $\rho\left(I-\alpha\left(B A^{-1} B^{T}+C\right)\right)$ as a measure of the effectiveness of the algorithm, and refer to it as the convergence factor. With respect to this measure, the optimal rate of convergence is achieved with the choice of $\alpha$ for which the algebraically smallest and largest eigenvalues of $I-\alpha\left(B A^{-1} B^{T}+C\right)$ are equal in absolute value. This gives

$$
\alpha=2 /\left(\lambda_{1}+\lambda_{2}\right)
$$

and for this choice, we have

$$
\rho\left(I-\alpha\left(B A^{-1} B^{T}+C\right)\right)=(\kappa-1) /(\kappa+1),
$$

where $\kappa=\lambda_{2} / \lambda_{1}$ is the condition number of $B A^{-1} B^{T}+C$.

Each step of the Uzawa algorithm requires the solution of a linear system with coefficient matrix $A$. Our primary concern in this paper is to show that if this system is not solved exactly, then the resulting "inexact" Uzawa algorithm can also be made to be convergent. In particular, we derive bounds on the rate of convergence that show that with relatively modest requirements on the accuracy of the approximate solution, the rate of convergence of the inexact algorithm is close to that of the exact version. Thus, if the linear solve is expensive, an approximate solution obtained by an "inner iteration" can be used without significant degradation of the outer iteration. The analysis is independent of the method used for the inner iteration. In addition, we show that preconditioning can be used with the inexact Uzawa method, and essentially the same convergence analysis applies.

The use of an approximate "inner" system solution has been considered in the context of preconditioners for iterative methods for linear systems (see [17] and references therein) and to develop inexact Newton methods for nonlinear systems [11]. The results presented here answer a long-standing question concerning the possibility of using inexact solutions for the Uzawa algorithm. For example, for solving the discrete Stokes equations (see §4), Fortin and Glowinski [13, pp. 66ff] empirically document the effect of using a fixed number of successive overrelaxation (SOR) steps for the inner solve, observing that a relatively small number (on the order of 10) steps is needed to reproduce the convergence rate of the exact algorithm. Inexact solutions to the Poisson equation have also been incorporated into other solution algorithms for the Stokes equations. Our approach is in some ways related to that of Verfürth [29], who uses the conjugate gradient method (CG) to solve (4). It is well known that CG displays faster convergence than what (7) provides, but for (4), it requires an accurate computation of the action of $A^{-1}$. The method of [29] avoids this issue 
by applying CG to a modification of (4) in which $A^{-1}$ is replaced by an approximation, $M$; the accuracy of the resulting solution as an approximation to the solution of the differential equation is a function of the accuracy with which $M$ approximates $A^{-1}$. Several other methods have been developed that replace exact Poisson solutions with preconditioners applied to the complete system (1); see Bramble and Pasciak [5], Bank, Welfert and Yserentant [4], Rusten and Winther [25], and Silvester and Wathen [27], [30]. Cf. also Queck [24] for an analysis of the Arrow-Hurwicz algorithm, which can be viewed as an inexact Uzawa method. The recent report by Welfert [33] gives additional analysis of the inexact Uzawa algorithm.

An outline of the rest of the paper is as follows. In $\S 2$, we present the inexact Uzawa algorithm and the analysis of its convergence, and in $\S 3$, we show how the results extend to handling preconditioned problems. We will use the discrete Stokes equations as a model problem, and in $\S 4$, we outline some properties of this example. Finally, in $\S 5$, we present the results of numerical experiments that demonstrate the effectiveness of the ideas developed here.

2. The inexact Uzawa algorithm. The inexact version of the Uzawa algorithm starts with an initial guess $p_{0}$ and performs the following iteration:

$$
\begin{aligned}
& \text { for } k=0 \text { until convergence, do } \\
& \text { Compute } u_{k+1} \text { such that } A u_{k+1}=f-B^{T} p_{k}+\delta_{k} \\
& \quad \text { Compute } p_{k+1}=p_{k}+\alpha\left(B u_{k+1}-C p_{k}\right) \\
& \text { enddo }
\end{aligned}
$$

The vector $\delta_{k}$ is the residual of the approximate solution $u_{k+1}$ to the system $A v=$ $f-B^{T} p_{k}$. The magnitude of $\delta_{k}$ depends on the stopping criterion used with the iterative method applied to this system. As we will show below, a natural choice is

$$
\left\|\delta_{k}\right\|_{2}<\tau\left\|B u_{k}-C p_{k-1}\right\|_{2},
$$

for $k \geq 1$, where $\tau$ is a parameter. Note that the quantity $B u_{k}-C p_{k-1}$ appearing in the right-hand side of this expression is the residual of the second block row of (1) for the approximate solution pair $\left(u_{k}, p_{k-1}\right)$; this quantity has already been computed for the update of $p_{k}$ in the previous step. Moreover, let $e_{k}=u-u_{k}$ and $\epsilon_{k}=p-p_{k}$ denote the error vectors. From (1), the right side of (9) is equal to $\tau\left\|B e_{k}-C \epsilon_{k-1}\right\|_{2}$.

Before considering the inexact algorithm, we derive one result for the exact version that shows that there is a bound analogous to (5) involving the errors $e_{k}$.

LemMA 2.1. For $k \geq 1$, the errors associated with the Uzawa algorithm (2) satisfy

$$
\left(B e_{k+1}-C \epsilon_{k}\right)=\left[I-\alpha\left(B A^{-1} B^{T}+C\right)\right]\left(B e_{k}-C \epsilon_{k-1}\right),
$$

and therefore

$$
\left\|B e_{k+1}-C \epsilon_{k}\right\|_{2} \leq\left[\rho\left(I-\alpha\left(B A^{-1} B^{T}+C\right)\right)\right]^{k}\left\|B e_{1}-C \epsilon_{0}\right\|_{2} .
$$

Proof. It follows from the definition of $u_{k+1}$ and $p_{k+1}$ in (2) that

$$
A\left(u_{k}-u_{k+1}\right)=B^{T}\left(p_{k}-p_{k-1}\right)=\alpha B^{T} B u_{k}-\alpha B^{T} C p_{k-1} \quad \text { for } k \geq 1 .
$$

Consequently,

$$
e_{k+1}=e_{k}+\alpha A^{-1} B B^{T} u_{k}-\alpha A^{-1} B^{T} C p_{k-1} \text {. }
$$


Subtracting $0=\alpha A^{-1} B^{T} B u-\alpha A^{-1} B^{T} C p$ from the right side of this expression and then premultiplying both sides by $B$ gives

$$
B e_{k+1}=B e_{k}-\alpha B A^{-1} B^{T} B e_{k}+\alpha B A^{-1} B^{T} C \epsilon_{k-1} .
$$

It also follows from (1) and the definition of $p_{k+1}$ that

$$
C \epsilon_{k}=C \epsilon_{k-1}-\alpha C\left(C \epsilon_{k-1}\right)+\alpha C B e_{k} .
$$

Subtracting (13) from (12) yields (10).

Thus, $\rho\left(I-\alpha\left(B A^{-1} B^{T}+C\right)\right)$ also governs the convergence of the quantities $\left\{B e_{k+1}-C \epsilon_{k}\right\}$. This result for the case $C=0$ can be found in [13, pp. 8ff.].

In the inexact version, the iterates $\left\{u_{k}\right\}$ satisfy

$$
\begin{aligned}
A u_{k+1} & =f-B^{T} p_{k}+\delta_{k}, \\
A u_{k} & =f-B^{T} p_{k-1}+\delta_{k-1},
\end{aligned}
$$

so that

$$
A\left(u_{k}-u_{k+1}\right)=\alpha B^{T} B u_{k}-\alpha B^{T} C p_{k-1}-\delta_{k}+\delta_{k-1} .
$$

Thus, exactly as in the proof of Lemma 2.1 , we have for $k \geq 1$

$$
\left(B e_{k+1}-C \epsilon_{k}\right)=\left[I-\alpha\left(B A^{-1} B^{T}+C\right)\right]\left(B e_{k}-C \epsilon_{k-1}\right)-B A^{-1} \delta_{k}+B A^{-1} \delta_{k-1} .
$$

These quantities are bounded in norm by

$$
\begin{aligned}
\left\|B e_{k+1}-C \epsilon_{k}\right\|_{2} \leq & \left\|I-\alpha\left(B A^{-1} B^{T}+C\right)\right\|_{2}\left\|B e_{k}-C \epsilon_{k-1}\right\|_{2} \\
& +\left\|B A^{-1}\right\|_{2}\left(\left\|\delta_{k}\right\|_{2}+\left\|\delta_{k-1}\right\|_{2}\right) \\
\leq & \left\|I-\alpha\left(B A^{-1} B^{T}+C\right)\right\|_{2}\left\|B e_{k}-C \epsilon_{k-1}\right\|_{2} \\
& +\tau\left\|B A^{-1}\right\|_{2}\left(\left\|B e_{k}-C \epsilon_{k-1}\right\|_{2}+\left\|B e_{k-1}-C \epsilon_{k-2}\right\|_{2}\right),
\end{aligned}
$$

where the second inequality is a consequence of the choice of stopping criterion (9), and it holds only for $k \geq 2$.

Now, let us adopt the notation

$$
\rho=\left\|I-\alpha\left(B A^{-1} B^{T}+C\right)\right\|_{2}, \quad \eta=\tau\left\|B A^{-1}\right\|_{2} .
$$

Our motivation for the criterion (9) is that from (14), $\left\|B e_{k}-C \epsilon_{k-1}\right\|_{2}$ is bounded by $\beta_{k}$, the solution to the three-term recurrence

$$
\beta_{k+1}=\rho \beta_{k}+\eta\left(\beta_{k}+\beta_{k-1}\right) \text { for } k \geq 2,
$$

where $\beta_{1}=\left\|B e_{1}-C \epsilon_{0}\right\|_{2}$ and $\beta_{2}=\left\|B e_{2}-C \epsilon_{1}\right\|_{2}$ are given. The solution is

$$
\beta_{k}=a_{1} r_{+}^{k}+a_{2} r_{-}^{k}
$$

where

$$
r_{ \pm}=\frac{\rho+\eta}{2}\left(1 \pm \sqrt{1+4 \eta /(\rho+\eta)^{2}}\right) .
$$

The coefficients are given by

$$
a_{1}=\frac{2\left(\beta_{2}-\beta_{1} r_{-}\right)}{(\rho+\eta)^{2} s(s+1)}, \quad a_{2}=\frac{2\left(\beta_{2}-\beta_{1} r_{+}\right)}{(\rho+\eta)^{2} s(s-1)},
$$


where $s=\sqrt{1+4 \eta /(\rho+\eta)^{2}}$. Thus, $r_{+}$and $a_{1}$ are greater than zero, and $r_{-}$is less than zero. More importantly, we have

$$
\left|\beta_{k}\right| \leq \max \left(a_{1},\left|a_{2}\right|\right)\left(r_{+}^{k}+\left|r_{-}\right|^{k}\right)<2 \max \left(a_{1},\left|a_{2}\right|\right) r_{+}^{k} .
$$

Let $c=\max \left(a_{1},\left|a_{2}\right|\right)$. Expressing $r_{+}$in slightly different form, we have the following result.

THEOREM 2.2. The errors generated by the inexact Uzawa algorithm satisfy

$$
\left\|B e_{k}-C \epsilon_{k-1}\right\|_{2} \leq c \hat{\rho}^{k}
$$

for $k \geq 2$, where

$$
\hat{\rho}=r_{+}=\rho+\eta+\frac{1}{2}(\rho+\eta)\left(\sqrt{1+4 \eta /(\rho+\eta)^{2}}-1\right),
$$

and the constant $c$ is independent of $k$.

Comparison with (11) shows that the quantity

$$
\eta+\frac{1}{2}(\rho+\eta)\left(\sqrt{1+4 \eta /(\rho+\eta)^{2}}-1\right)
$$

is a bound on the degradation of performance associated with inexact computation of $A^{-1}\left(f-B^{T} p_{k}\right)$. In the limit as $\tau \rightarrow 0$, this perturbation is approximately $\eta(1+1 /(\rho+$ $\eta)$ ), and from the definition of $\eta$ in (15), it is evident that the perturbation tends to zero with $\tau$. We will examine the extent to which the bound predicts performance in $\S 5$.

Although Theorem 2.2 applies only to the quantities $\left\{B e_{k}-C \epsilon_{k-1}\right\}$, we now show that this result can be used to derive bounds for both errors $e_{k}$ and $\epsilon_{k}$ leading to the same convergence factors. It follows from (8) that

$$
p_{k+1}=p_{k}+\alpha B A^{-1}\left(f-B^{T} p_{k}\right)+\alpha B A^{-1} \delta_{k},
$$

for all $k \geq 0$, so that

$$
\epsilon_{k+1}=\left[I-\alpha\left(B A^{-1} B^{T}+C\right)\right] \epsilon_{k}-\alpha B A^{-1} \delta_{k} .
$$

Therefore,

$$
\begin{aligned}
& \left\|\epsilon_{k+1}\right\|_{2} \leq \rho\left\|\epsilon_{k}\right\|+\alpha \tau\left\|B A^{-1}\right\|_{2}\left\|B e_{k}-C \epsilon_{k-1}\right\|_{2} \quad \text { for } k \geq 1, \quad \text { by (9), } \\
& \leq \rho\left\|\epsilon_{k}\right\|+\alpha \tau c\left\|B A^{-1}\right\|_{2} \hat{\rho}^{k} \quad \text { for } k \geq 2 \text {, by Theorem 2.2. }
\end{aligned}
$$

We rewrite the second inequality concisely as

$$
\left\|\epsilon_{k}\right\|_{2} \leq \rho\left\|\epsilon_{k-1}\right\|+\hat{\eta} \hat{\rho}^{k-1}
$$

for $k \geq 3$, where $\hat{\eta}=\alpha \tau c\left\|B A^{-1}\right\|_{2}$. Applying this result recursively gives the bound

$$
\left\|\epsilon_{k}\right\|_{2} \leq \rho^{k-2}\left\|\epsilon_{2}\right\|_{2}+\hat{\eta} \sum_{j=0}^{k-3} \rho^{j} \hat{\rho}^{k-1-j} .
$$

But

$$
\sum_{j=0}^{k-3} \rho^{j} \hat{\rho}^{k-1-j}=\hat{\rho}^{k-1} \sum_{j=0}^{k-3}\left(\frac{\rho}{\hat{\rho}}\right)^{j}=\hat{\rho}^{k-1}\left(\frac{1-(\rho / \hat{\rho})^{k-2}}{1-\rho / \hat{\rho}}\right)<\frac{\hat{\rho}^{k}}{\hat{\rho}-\rho}
$$


Combining this with (17) gives the following result.

COROLlary 2.3. The errors $\epsilon_{k}=p-p_{k}$ for the inexact Uzawa algorithm satisfy

$$
\left\|\epsilon_{k}\right\|_{2}<\rho^{k-2}\left\|\epsilon_{2}\right\|_{2}+\hat{c} \hat{\rho}^{k}
$$

for $k \geq 3$, where $\hat{c}=\alpha \tau c\left\|B A^{-1}\right\|_{2} /(\hat{\rho}-\rho)$ is independent of $k$.

As $k$ increases, this bound is dominated by $\hat{\rho}^{k}$, so that $\hat{\rho}$ is a bound on the convergence factor associated with $\left\{p_{k}\right\}$.

Finally, we can use Theorem 2.2 and Corollary 2.3 to derive a bound on the norms of the errors $e_{k}$. We will consider both the energy norm $\left\|e_{k}\right\|_{A}=\left(e_{k}, A e_{k}\right)^{1 / 2}$ and the Euclidean norm $\left\|e_{k}\right\|_{2}$. For the former, we will assume, as is true for finite element discretizations of the Stokes equations (see [29]), that there exists a positive definite matrix $M$ and positive $\Gamma$ such that

$$
\left|\left(v, B^{T} q\right)\right| \leq \Gamma\|v\|_{A}(q, M q)^{1 / 2}
$$

COROLlaRY 2.4. The errors $e_{k}=u-u_{k}$ for the inexact Uzawa algorithm satisfy

$$
\left\|e_{k}\right\|_{A}<\tilde{c}_{1} \hat{\rho}^{k}, \quad\left\|e_{k}\right\|_{2}<\tilde{c}_{2} \hat{\rho}^{k}
$$

for $k \geq 3$, where $\tilde{c}_{1}$ and $\tilde{c}_{2}$ are independent of $k$.

Proof. From (1) and the definition of $u_{k+1}$ in (8), we have

$$
A e_{k+1}=-B^{T} \epsilon_{k}-\delta_{k}
$$

First, consider the energy norm. The errors in the velocities satisfy

$$
\left\|e_{k+1}\right\|_{A}^{2}=\left(e_{k+1}, A e_{k+1}\right)=-\left(e_{k+1}, B^{T} \epsilon_{k}\right)-\left(e_{k+1}, \delta_{k}\right) .
$$

Using (18) and Corollary 2.3, the first term is bounded by

$$
\begin{aligned}
\left|\left(e_{k+1}, B^{T} \epsilon_{k}\right)\right| & \leq \Gamma\left\|e_{k+1}\right\|_{A} \lambda_{\max }(M)^{1 / 2}\left\|\epsilon_{k}\right\|_{2} \\
& \leq \Gamma\left\|e_{k+1}\right\|_{A} \lambda_{\max }(M)^{1 / 2} \tilde{c} \hat{\rho}^{k}
\end{aligned}
$$

where $\tilde{c}$ is independent of $k$. By the Cauchy-Schwarz inequality, (9), and Theorem 2.2 , the second term satisfies

$$
\begin{aligned}
\left|\left(e_{k+1}, \delta_{k}\right)\right| & \leq\left\|e_{k+1}\right\|_{A}\left\|A^{-1} \delta_{k}\right\|_{A} \leq\left\|e_{k+1}\right\|_{A} \lambda_{\max }\left(A^{-1}\right)\left\|\delta_{k}\right\|_{2} \\
& \leq\left\|e_{k+1}\right\|_{A} \lambda_{\max }\left(A^{-1}\right) c \tau \hat{\rho}^{k}
\end{aligned}
$$

The bound for the energy norm follows from (20)-(22).

For the Euclidean norm, we premultiply (19) by $A^{-1}$ and use (9), which gives

$$
\left\|e_{k+1}\right\|_{2} \leq\left\|A^{-1} B^{T}\right\|_{2}\left\|\epsilon_{k}\right\|_{2}+\tau\left\|A^{-1}\right\|_{2}\left\|B e_{k}-C \epsilon_{k-1}\right\|_{2}
$$

The first term is bounded by Corollary 2.3, and the second by Theorem 2.2.

Remark 2.1. A bound for $\left\|e_{k}\right\|_{2}$ could be derived directly from the bound on the energy norm; the argument above avoids reference to (18). 
3. Preconditioning. Preconditioned exact and inexact Uzawa algorithms are defined as follows. Let $Q=L L^{T}$ denote a symmetric positive-definite matrix of the same order as $B A^{-1} B^{T}$. Formally, a preconditioned version of (4) with $Q$ as a preconditioner is given by

$$
\left[L^{-1}\left(B A^{-1} B^{T}+C\right) L^{-T}\right]\left[L^{T} p\right]=\left[L^{-1} B A^{-1} f\right] .
$$

This is precisely the Schur complement for the preconditioned version of (1) given by

$$
\left[\left(\begin{array}{ll}
I & 0 \\
0 & L^{-1}
\end{array}\right)\left(\begin{array}{ll}
A & B^{T} \\
B & -C
\end{array}\right)\left(\begin{array}{ll}
I & 0 \\
0 & L^{-T}
\end{array}\right)\right]\left[\left(\begin{array}{c}
u \\
L^{T} p
\end{array}\right)\right]=\left(\begin{array}{l}
f \\
0
\end{array}\right),
$$

or, in compact form,

$$
\left(\begin{array}{cc}
A & \tilde{B}^{T} \\
\tilde{B} & -\tilde{C}
\end{array}\right)\left(\begin{array}{c}
u \\
\tilde{p}
\end{array}\right)=\left(\begin{array}{l}
f \\
0
\end{array}\right)
$$

where $\tilde{B}=L^{-1} B, \tilde{C}=L^{-1} C L^{-T}$, and $\tilde{p}=L^{T} p$. Formally applying the Uzawa algorithm to the system (24) would produce a set of approximations $\tilde{p}_{k}$ to $\tilde{p}$. Letting $p_{k}=L^{-T} \tilde{p}_{k}$ leads to the preconditioned Uzawa algorithm:

$$
\begin{aligned}
& \text { for } k=0 \text { until convergence, do } \\
& \quad \text { Solve } A u_{k+1}=f-B^{T} p_{k} \\
& \quad \text { Compute } p_{k+1}=p_{k}+\alpha Q^{-1}\left(B u_{k+1}-C p_{k}\right) \\
& \text { enddo }
\end{aligned}
$$

Each iteration requires the computation of a preconditioning solve of the form $Q w=v$ for $w$.

The inexact version of the preconditioned Uzawa algorithm is defined exactly as in (8): $u_{k+1}$ is chosen so that $A u_{k+1}=f-B^{T} p_{k}+\delta_{k}$. The natural generalization of the stopping criterion (9) is

$$
\left\|\delta_{k}\right\|_{2}<\tau\left\|B u_{k}-C p_{k-1}\right\|_{Q^{-1}} .
$$

The vector $Q^{-1}\left(B u_{k}-C p_{k-1}\right)$ needed here is available from the computation of $p_{k}$.

Let $\rho_{Q}=\rho\left(I-\alpha\left(\tilde{B} A^{-1} \tilde{B}^{T}+\tilde{C}\right)\right)$. The analysis of both the exact and inexact Uzawa algorithms applies to the preconditioned versions essentially verbatim, except that norms depending on $Q$ are involved. In particular, straightforward algebraic manipulations give the following analogues of (5), (11), Theorem 2.2, and Corollaries 2.3 and 2.4 .

THEOREM 3.1. The errors associated with the preconditioned exact Uzawa algorithm satisfy

$$
\left\|\epsilon_{k}\right\|_{Q} \leq \rho_{Q}^{k}\left\|\epsilon_{0}\right\|_{Q}, \quad\left\|B e_{k+1}-C \epsilon_{k}\right\|_{Q^{-1}} \leq \rho_{Q}^{k}\left\|B e_{1}-C \epsilon_{0}\right\|_{Q^{-1}}
$$

The errors associated with the preconditioned inexact Uzawa algorithm with stopping criterion (25) satisfy

$$
\begin{array}{ll}
\left\|B e_{k}-C \epsilon_{k-1}\right\|_{Q^{-1}} \leq c_{Q} \hat{\rho}_{Q}^{k} & \text { for } k \geq 2, \\
\left\|\epsilon_{k}\right\|_{Q}<\rho_{Q}^{k-2}\left\|\epsilon_{2}\right\|_{Q}+\hat{c}_{Q} \hat{\rho}_{Q}^{k} & \text { for } k \geq 3, \\
\left\|e_{k}\right\|_{A}<\tilde{c}_{Q, 1} \hat{\rho}_{Q}^{k}, \quad\left\|e_{k}\right\|_{2}<\tilde{c}_{Q, 2} \hat{\rho}_{Q}^{k} & \text { for } k \geq 3,
\end{array}
$$


where $\hat{\rho}_{Q}$ is defined as in (16),

$$
\hat{\rho}_{Q}=\rho_{Q}+\eta_{Q}+\frac{1}{2}\left(\rho_{Q}+\eta_{Q}\right)\left(\sqrt{1+4 \eta_{Q}\left(\rho_{Q}+\eta_{Q}\right)^{2}}-1\right),
$$

and $\eta_{Q}=\tau\left\|\tilde{B} A^{-1}\right\|_{2}$.

The constants $c_{Q}, \hat{c}_{Q}, \tilde{c}_{Q, 1}$, and $\tilde{c}_{Q, 2}$ arise exactly as in $\S 2$; we omit the details.

4. Model problem: The discrete Stokes equations. We will use the Stokes equations

$$
\begin{array}{ll}
-\Delta u+\nabla p=f & \text { on } \Omega=(0,1) \times(0,1), \\
-\operatorname{div} u=0 & \text { on } \partial \Omega, \\
u=0 & \\
\int_{\Omega} p=0 &
\end{array}
$$

to illustrate and supplement the analysis. In this section, we review some basic facts about finite element discretization of this problem and outline some numerical properties of two specific discretizations. See [8], [15], [16], and [19] for more general discussions of this issue.

Remark 4.1. We are using this example to compare the analytic results of $\S \S 2-$ 3 with experimental performance. We note that a variety of methods have been proposed for solving discretizations of (26), including applications of the conjugate gradient method to symmetric positive-definite reformulations of (1) [4], [5], [29]; the conjugate residual method applied directly to the indefinite system [25], [27], [30]; and multigrid methods applied directly to (1); see, e.g., [6], [22], and [34]. We expect some of these methods to converge more rapidly than the Uzawa method for the discrete Stokes equations. A detailed comparison of these ideas is beyond the scope of this paper.

Let $H_{0}^{1}(\Omega)$ denote the Sobelev space of functions satisfying homogeneous Dirichlet boundary conditions, and whose first derivatives are in $L^{2}(\Omega)$; let $L_{0}^{2}(\Omega)=\{\psi \in$ $\left.L^{2}(\Omega) \mid \int_{\Omega} \psi=0\right\}$; and let $V_{h} \subset H_{0}^{1}(\Omega) \times H_{0}^{1}(\Omega), P_{h} \subset L_{0}^{2}(\Omega)$ denote finite-dimensional subspaces. In addition, let $C_{h}$ denote a continuous symmetric positive-semidefinite bilinear form defined on $P_{h} \times P_{h}$. The finite element solution to (26) is the unique pair $\left(u_{h}, p_{h}\right) \in V_{h} \times P_{h}$ such that

$$
\begin{array}{ll}
\left(\operatorname{grad} u_{h}, \operatorname{grad} \phi\right)-\left(p_{h}, \operatorname{div} \phi\right)=(f, \phi) & \text { for all } \phi \in V_{h}, \\
-\left(\psi, \operatorname{div} u_{h}\right)-C_{h}\left(p_{h}, \psi\right)=0 & \text { for all } \psi \in P_{h},
\end{array}
$$

where (, ) represents the $L^{2}$ inner product in $H_{0}^{1}(\Omega) \times H_{0}^{1}(\Omega)$ or $L_{0}^{2}(\Omega)$, as appropriate. Let $u_{h}=\sum_{i} u_{i} \phi_{i}$ and $p_{h}=\sum_{j} p_{j} \psi_{j}$, where $\left\{\phi_{i}\right\}$ and $\left\{\psi_{i}\right\}$ are bases for $V_{h}$ and $P_{h}$, respectively. In matrix notation, (27) has the form (1), where $A$ is the global stiffness matrix $\left[\left(\operatorname{grad} \phi_{i}, \operatorname{grad} \phi_{j}\right)\right], B$ is the Gram matrix $\left[\left(\phi_{i}, \operatorname{div} \psi_{j}\right)\right], C$ is the Gram matrix $\left[C_{h}\left(\psi_{i}, \psi_{j}\right)\right]$, and $\phi_{i}, \phi_{j}$ and $\psi_{i}, \psi_{j}$ range over the bases for $V_{h}$ and $P_{h}$. In addition, let $M=\left[\left(\psi_{i}, \psi_{j}\right)\right]$ denote the global mass matrix associated with the pressure discretization.

We assume that the discretization satisfies

$$
\gamma^{2} \leq \frac{\left(q,\left(B A^{-1} B^{T}+C\right) q\right)}{(q, M q)} \leq \Gamma^{2}
$$

where $\gamma$ and $\Gamma$ are independent of $h$. In the case $C=0$, the lower bound holds for discretizations satisfying the inf-sup condition

$$
\inf _{q \in P_{h}} \sup _{v \in V_{h}} \frac{\left(v, B^{T} q\right)}{(v, A v)^{1 / 2}(q, M q)^{1 / 2}} \geq \gamma,
$$


and the condition (18) holds with $\Gamma \leq \sqrt{d}$; see, e.g., [3], [7], [29], and [30]. The upper bound follows from this. The purpose of the stabilization matrix $C$ is to impose (28) in cases where (29) is not satisfied; in this case, the value of $\Gamma$ in (28) will be larger than that derived from (18) [9], [26].

We will consider two discretizations of $(26)$, on uniform triangular meshes:

1. $P_{1}(h) P_{1}(2 h)$ : continuous piecewise linear velocities on a mesh of width $h$, and continuous piecewise linear pressures on a mesh of width $2 h$;

2. Stabilized $P_{1}(h) P_{1}(h)$ : continuous piecewise linear velocities and pressures on a mesh of width $h$, together with the stabilization matrix $C=\beta h^{2} A_{n}$, where $A_{n}$ is a discrete Laplace operator defined on the pressure space, subject to Neumann boundary conditions [9].

The first choice satisfies the inf-sup condition. The second one is equivalent to the mini-element discretization [1] after elimination of the internal degrees of freedom, and it satisfies (28) with $\Gamma$ dependent on $\beta$ [26]. The usual hat functions are used as the basis for both velocities and pressures. ${ }^{1}$ For stabilized $P_{1}(h) P_{1}(h)$, we use $\beta=.025$, as recommended in [26]. In tables of experimental results shown below, we refer to this discretization simply as " $P_{1}(h) P_{1}(h)$."

It follows from $(28)$ that for $q \neq 0$,

$$
\gamma^{2} \mu_{1} \leq \frac{\left(q,\left(B A^{-1} B^{T}+C\right) q\right)}{(q, q)} \leq \Gamma^{2} \mu_{2},
$$

where $\mu_{1}$ and $\mu_{2}$ are the smallest and largest eigenvalues, respectively, of $M$. Therefore, the condition number $\kappa$ of $B A^{-1} B^{T}+C$ is bounded above by

$$
\kappa \leq\left(\Gamma^{2} / \gamma^{2}\right)\left(\mu_{2} / \mu_{1}\right)
$$

In light of (7), it is desirable to have $\kappa$ as small as possible. The condition numbers for these discretizations with a uniform triangulation of $\Omega=(0,1) \times(0,1)$ on a mesh of width $h=1 / 32$ are shown in Table 1 (with preconditioning "None"). The table also shows the bound (31), computed values of the quantities $\Gamma^{2} / \gamma^{2}$ and $\mu_{2} / \mu_{1}$, and the convergence factor $\rho\left(I-\alpha\left(B A^{-1} B^{T}+C\right)\right)$. The bound (31) is qualitatively similar to the condition number for the $P_{1}(h) P_{1}(2 h)$ discretization, but it is pessimistic for stabilized $P_{1}(h) P_{1}(h)$.

Remark 4.2. For the bases used for these discretizations, the mass matrix has the form $M=h^{2} \hat{M}$, where both the magnitudes of the nonzero entries and the extreme eigenvalues of $\hat{M}$ are independent of $h$ [14]. Consequently, from (30), the optimal value of $\alpha$ is of magnitude $O\left(h^{-2}\right)$.

Remark 4.3. It is also shown in [14] that the condition number $\mu_{2} / \mu_{1}$ of the mass matrix can be bounded by $n_{e}\left(\mu_{2, e} / \mu_{1, e}\right)$, where $\mu_{1, e}, \mu_{2, e}$ are the smallest, respectively, largest eigenvalues of the element mass matrices, and $n_{e}$ is the maximum number of elements around any nodal point. For the discretizations under consideration, this bound is 24 , somewhat larger than the computed value of 14.50 .

The convergence factors can be reduced by preconditioning. The extreme eigenvalues of the preconditioned matrix $\tilde{B} A^{-1} \tilde{B}^{T}+\tilde{C}$ are given by the smallest and largest

\footnotetext{
1 With these bases, the matrix $B$ is rank deficient. For the discretizations under consideration, the coefficient matrices of (1) and the Schur complements $B A^{-1} B^{T}+C$ are also rank deficient, with deficiency 1. The condition numbers discussed below correspond to the ratio of the largest eigenvalue to the smallest nonzero eigenvalue.
} 
TABLE 1

Condition numbers, bounds, and convergence factors for $h=1 / 32$.

\begin{tabular}{ll|rr|rr|c} 
Discretization & Preconditioning & \multicolumn{1}{|c}{$\kappa$} & Bound & $\Gamma^{2} / \gamma^{2}$ & $\mu_{2} / \mu_{1}$ & $\rho$ \\
\hline$P_{1}(h) P_{1}(2 h)$ & None & 128.07 & 145.82 & 10.06 & 14.50 & 0.985 \\
$P_{1}(h) P_{1}(2 h)$ & Diagonal & 22.71 & 40.22 & 10.06 & 4.00 & 0.916 \\
$P_{1}(h) P_{1}(2 h)$ & Tridiagonal & 16.19 & 30.28 & 10.06 & 3.01 & 0.884 \\
$P_{1}(h) P_{1}(h)$ & None & 26.15 & 101.49 & 6.94 & 14.50 & 0.926 \\
$P_{1}(h) P_{1}(h)$ & Diagonal & 9.82 & 27.76 & 6.94 & 4.00 & 0.815 \\
$P_{1}(h) P_{1}(h)$ & Tridiagonal & 9.86 & 20.89 & 6.94 & 3.01 & 0.816
\end{tabular}

values of the Rayleigh quotient

$$
\frac{\left(\tilde{q},\left(\tilde{B} A^{-1} \tilde{B}^{T}+\tilde{C}\right) \tilde{q}\right)}{(\tilde{q}, \tilde{q})}=\frac{\left(q,\left(B A^{-1} B^{T}+C\right) q\right)}{(q, Q q)}=\frac{\left(q,\left(B A^{-1} B^{T}+C\right) q\right)}{(q, M q)} \frac{(q, M q)}{(q, Q q)} .
$$

Consequently, as in (31), we have the bound

$$
\kappa \leq\left(\Gamma^{2} / \gamma^{2}\right)\left(\tilde{\mu}_{2} / \tilde{\mu}_{1}\right)
$$

where $\tilde{\kappa}$ is the condition number of $\tilde{B} A^{-1} \tilde{B}^{T}+\tilde{C}$, and $\tilde{\mu}_{1}$ and $\tilde{\mu}_{2}$ are the smallest and largest eigenvalues, respectively, of $Q^{-1} M$.

We consider two preconditioners derived from a result due to Wathen [31]. This idea has been used by Silvester and Wathen [27], [30] in the context of conjugate residual methods. Cf. [10] for other techniques developed for time-dependent problems. The mass matrix $M$ can be expressed as a sum of local element matrices $\sum_{e} L_{e}^{T} M_{e} L_{e}$, where $M_{e}$ is the local element matrix associated with element $e$, and $L_{e}$ is a Boolean matrix that maps global unknowns into local ones. Let $Q_{e}$ denote a nonsingular symmetric matrix of the same order as $M_{e}$ such that $Q=\sum_{e} L_{e}^{T} Q_{e} L_{e}$ is symmetric positive definite. Then

$$
\min _{e} \min _{q_{e}} \frac{\left(q_{e}, M_{e} q_{e}\right)}{\left(q_{e}, Q_{e} q_{e}\right)} \leq \frac{(q, M q)}{(q, Q q)} \leq \max _{e} \max _{q_{e}} \frac{\left(q_{e}, M_{e} q_{e}\right)}{\left(q_{e}, Q_{e} q_{e}\right)} .
$$

That is, the extreme eigenvalues of $Q^{-1} M$ are bounded by those of the preconditioned local matrices $Q_{e}^{-1} M_{e}$. Wathen shows that for the choice of $Q_{e}=\operatorname{diag}\left(M_{e}\right)$, producing $Q=\operatorname{diag}(M)$, the preconditioned condition number $\tilde{\mu}_{2} / \tilde{\mu}_{1}$ is small for a variety of discretizations, and it is bounded by 4 for piecewise linear pressures. It is also possible to express $Q=\operatorname{tridiag}(M)$, consisting of the three interior diagonals of $M$, as a sum of element matrices; see [32]. Using the techniques of [31], [32], it can be shown that in this case the preconditioned condition number is bounded by $2+\sqrt{3}$. Recalling that $M=h^{2} \hat{M}$, we will consider the two choices $Q=s_{Q} \operatorname{diag}(\hat{M})$ and $Q=s_{Q} \operatorname{tridiag}(\hat{M})$. Condition numbers, bounds and convergence factors for the preconditioned exact Uzawa algorithm are also shown in Table 1. We will discuss the scalings $s_{Q}$ below.

The bounds on the convergence factor for the inexact Uzawa algorithm also depend on $\left\|B A^{-1}\right\|_{2}=\left\|A^{-1} B^{T}\right\|_{2}$ (see (15)), or, for the preconditioned problem, on $\left\|\tilde{B} A^{-1}\right\|_{2}$ (see Theorem 3.1). But

$$
\begin{aligned}
\left\|A^{-1} B^{T}\right\|_{2}^{2} & =\max _{q \in P_{h}} \frac{\left(A^{-1} B^{T} q, A^{-1} B^{T} q\right)}{(q, q)} \\
& =\max _{q \in P_{h}} \frac{\left(A^{-1} B^{T} q, A^{-1} B^{T} q\right)}{\left(A^{-1} B^{T} q, B^{T} q\right)} \frac{\left(A^{-1} B^{T} q, B^{T} q\right)}{(q, q)} \\
& \leq \lambda_{\max }\left(A^{-1}\right) \lambda_{\max }\left(B A^{-1} B^{T}\right) .
\end{aligned}
$$


TABLE 2

Values of $\left\|B A^{-1}\right\|_{2}$ and bounds for $h=1 / 32$.

\begin{tabular}{ll|cc} 
Discretization & Preconditioning & $\left\|B A^{-1}\right\|_{2}$ & Bound \\
\hline$P_{1}(h) P_{1}(2 h)$ & None & 0.32 & 0.45 \\
$P_{1}(h) P_{1}(2 h)$ & Diagonal & 0.21 & 0.70 \\
$P_{1}(h) P_{1}(2 h)$ & Tridiagonal & 0.19 & 0.78 \\
$P_{1}(h) P_{1}(h)$ & None & 0.16 & 0.45 \\
$P_{1}(h) P_{1}(h)$ & Diagonal & 0.10 & 0.70 \\
$P_{1}(h) P_{1}(h)$ & Tridiagonal & 0.09 & 0.78
\end{tabular}

For the discretizations under consideration, $\lambda_{\max }\left(A^{-1}\right)=1 / \lambda_{\min }(A) \approx 1 /\left(2 \pi h^{2}\right)$, and, from (30) and Remarks 4.2 and $4.3, \lambda_{\max }\left(B A^{-1} B^{T}\right) \leq \Gamma^{2} \hat{\mu}_{2} h^{2}$, where $\hat{\mu}_{2}$ is independent of $h$. It follows that

$$
\left\|B A^{-1}\right\|_{2} \approx \sqrt{\leq} \Gamma \sqrt{\hat{\mu}_{2} /(2 \pi)}
$$

which is also independent of $h$. In the preconditioned case, we have $\left\|\tilde{B} A^{-1}\right\|_{2}=$ $\left\|L^{-1} B A^{-1}\right\|_{2}$, so that the scaling of $Q$ would appear to play a role. Because the bounds on the velocities in Theorem 3.1 contain terms derived from both $\left\|\epsilon_{k}\right\|_{Q}$ and $\left\|B e_{k}-C \epsilon_{k-1}\right\|_{Q^{-1}}$ (cf. (21)-(23)), we attempt to balance these contributions by choosing $s_{Q}$ to make $\lambda_{\max }(Q)=1 / \lambda_{\min }(Q)$. It is then straightforward to show that

$$
\left\|A^{-1} \tilde{B}^{T}\right\|_{2}^{2} \leq \lambda_{\max }\left(A^{-1}\right) \lambda_{\max }\left(B A^{-1} B^{T}\right) / \lambda_{\min }(Q),
$$

so that $\left\|A^{-1} \tilde{B}^{T}\right\|_{2}$ is also independent of $h$. Table 2 shows computed values and bounds for $\left\|B A^{-1}\right\|_{2}$ and $\left\|\tilde{B} A^{-1}\right\|_{2}$ for both discretizations and $h=1 / 32$. The bounds for the preconditioned problem are pessimistic, but in experiments we found performance to be completely insensitive to scaling, and we will not pursue this issue.

From these results, we see that the bounds $\hat{\rho}$ and $\hat{\rho}_{Q}^{k}$ on the convergence factors for the inexact Uzawa algorithm are independent of the mesh size $h$. However (for brevity, restricting our remarks to the unpreconditioned case), $\hat{c}$ of Corollary 2.3 contains $\alpha$ as a factor, and the terms defining $\tilde{c}_{1}$ and $\tilde{c}_{2}$ of Corollary 2.4 contain either $\alpha$ or $\lambda_{\max }\left(A^{-1}\right)$ as factors. From the discussion above, $\alpha$ and $\lambda_{\max }\left(A^{-1}\right)$ are of magnitude $O\left(h^{-2}\right)$ (see $\S 3$ ). Thus, although the coefficients of $\hat{\rho}$ in Corollaries 2.3 and 2.4 are independent of the iteration counter $k$, they are not independent of the mesh size. This does not affect asymptotic convergence factors as $k$ increases for fixed $h$. That is, if $d_{k}$ is a generic quantity representing any of the bounds on the error norms derived in this section, then $\lim _{k \rightarrow \infty}\left\|d_{k}\right\|^{1 / k} \leq \hat{\rho}$. We will present empirical results on convergence factors for the inexact algorithm in $\S 5$. The factor $h^{-2}$ does not appear in Theorem 2.2, or in the bounds for the exact method, where $\tau=0$.

5. Numerical experiments. We now discuss the performance of the inexact and preconditioned Uzawa algorithms for solving discrete versions of (26). The experiments were run on a Sun 630MP using Matlab 4.0. The right-hand side vector $f$ was a vector of random numbers uniformly distributed in $[-1,1] .^{2}$ The results correspond to the mesh size $h=1 / 32$; from other tests with $h=1 / 8$ and $1 / 16$, as well as a limited number of tests with smaller $h(=1 / 64)$, we observed that the values of all quantities were close to their asymptotic limits, and behavior for this mesh size

\footnotetext{
${ }^{2}$ As noted above, the coefficient matrices are rank deficient by one. As a result, the pressure solution is uniquely defined only up to an additive constant.
} 
TABLE 3

Comparison of asymptotic convergence factors and bounds for the iterates computed by the inexact Uzawa algorithm, for $h=1 / 32$.

\begin{tabular}{ll|cccc}
$P_{1}(h) P_{1}(2 h)$ & \multicolumn{1}{c|}{$\tau$} & SOR & MIC & MG & Bound \\
\cline { 2 - 5 } Diagonal preconditioner & 1 & 1.07 & 0.92 & 0.92 & 1.28 \\
$\rho=0.92$ & $1 / 4$ & 0.95 & 0.92 & 0.92 & 1.02 \\
$\rho_{e}=0.92$ & $1 / 16$ & 0.92 & 0.92 & 0.92 & 0.94 \\
& $1 / 64$ & 0.92 & 0.92 & 0.92 & 0.92 \\
& & & & & \\
$P_{1}(h) P_{1}(h)$ & $\tau$ & SOR & MIC & MG & Bound \\
Diagonal preconditioner & 1 & 0.93 & 0.79 & 0.79 & 1.02 \\
$\rho=0.82$ & $1 / 4$ & 0.82 & 0.80 & 0.79 & 0.88 \\
$\rho_{e}=0.80$ & $1 / 16$ & 0.80 & 0.80 & 0.80 & 0.83 \\
& $1 / 64$ & 0.80 & 0.80 & 0.80 & 0.82 \\
& & & & & \\
$P_{1}(h) P_{1}(2 h)$ & $\tau$ & SOR & MIC & MG & Bound \\
Tridiagonal preconditioner & 1 & 1.02 & 0.88 & 0.88 & 1.19 \\
$\rho=0.85$ & $1 / 4$ & 0.90 & 0.88 & 0.88 & 0.94 \\
$\rho_{e}=0.88$ & $1 / 16$ & 0.88 & 0.88 & 0.88 & 0.87 \\
& $1 / 64$ & 0.88 & 0.88 & 0.88 & 0.86 \\
$P_{1}(h) P_{1}(h)$ & & & & & \\
Tridiagonal preconditioner & 1 & 0.93 & 0.79 & 0.79 & 1.00 \\
$\rho=0.82$ & $1 / 4$ & 0.81 & 0.80 & 0.79 & 0.87 \\
$\rho_{e}=0.81$ & $1 / 16$ & 0.81 & 0.81 & 0.80 & 0.83 \\
& $1 / 64$ & 0.81 & 0.81 & 0.81 & 0.82
\end{tabular}

was representative. The outer iterations used the optimal values of $\alpha$ defined in (6), determined empirically. The initial guess was $p_{0}=0$, and the stopping criterion was

$$
\left\|r_{k}\right\|_{2} /\|f\|_{2}<10^{-6}
$$

where

$$
r_{k}=\left(\begin{array}{c}
f \\
0
\end{array}\right)-\left(\begin{array}{cc}
A & B^{T} \\
B & -C
\end{array}\right)\left(\begin{array}{l}
u_{k} \\
p_{k}
\end{array}\right)
$$

For the inexact Uzawa algorithm, three iterative methods were used to compute the approximate solution to $A u_{k+1}=f-B^{T} p_{k}$ : successive overrelaxation (SOR), with the optimal iteration parameter $\omega[28]$, [36]; the modified incomplete Cholesky preconditioned conjugate gradient method (MIC), with zero as the acceleration parameter [12], [20]; and multigrid (MG) with damped Jacobi relaxation and $\omega=2 / 3$ [23]. An iteration of multigrid consists of one complete $\mathrm{V}$-cycle with one presmoothing step and one postsmoothing step. The stopping criterion for the inner iteration was (9) or (25). For the first iteration, where $u_{0}$ is not defined, we used the stopping criterion $\left\|\delta_{0}\right\|_{2}<\tau\left\|f-B^{T} p_{0}\right\|_{2}$. To assess the accuracy of the solutions $\left(u_{k}, p_{k}\right)$ computed using (32), a more accurate solution $\left(u_{*}, p_{*}\right)$ was computed satisfying the criterion $\left\|r_{*}\right\|_{2} /\|f\|_{2}<10^{-12}$, and the differences $u_{*}-u_{k}$ and $p_{*}-p_{k}$ were used for the errors $e_{k}$ and $\epsilon_{k}$ in the velocities and pressures.

We first consider how well the convergence bounds of $\S 2$ predict performance. As noted in $\S 4$, the convergence factors for the error norms $\left\|\epsilon_{k}\right\|_{2},\left\|e_{k}\right\|_{2}$, and $\left\|e_{k}\right\|_{A}$ and the partial residual norm $\left\|B e_{k}-C \epsilon_{k-1}\right\|_{2}$ are all bounded by the same quantities, $\hat{\rho}$ or $\hat{\rho}_{Q}$. In our experiments, we observed that all these norms, as well as the residual norms $\left\|r_{k}\right\|_{2}$, displayed the same qualitive behavior. In the discussion below, we consider only one measure of the error, the commonly used norm $\zeta_{k}=\left\|e_{k}\right\|_{A}+\left\|\epsilon_{k}\right\|_{2}$. 

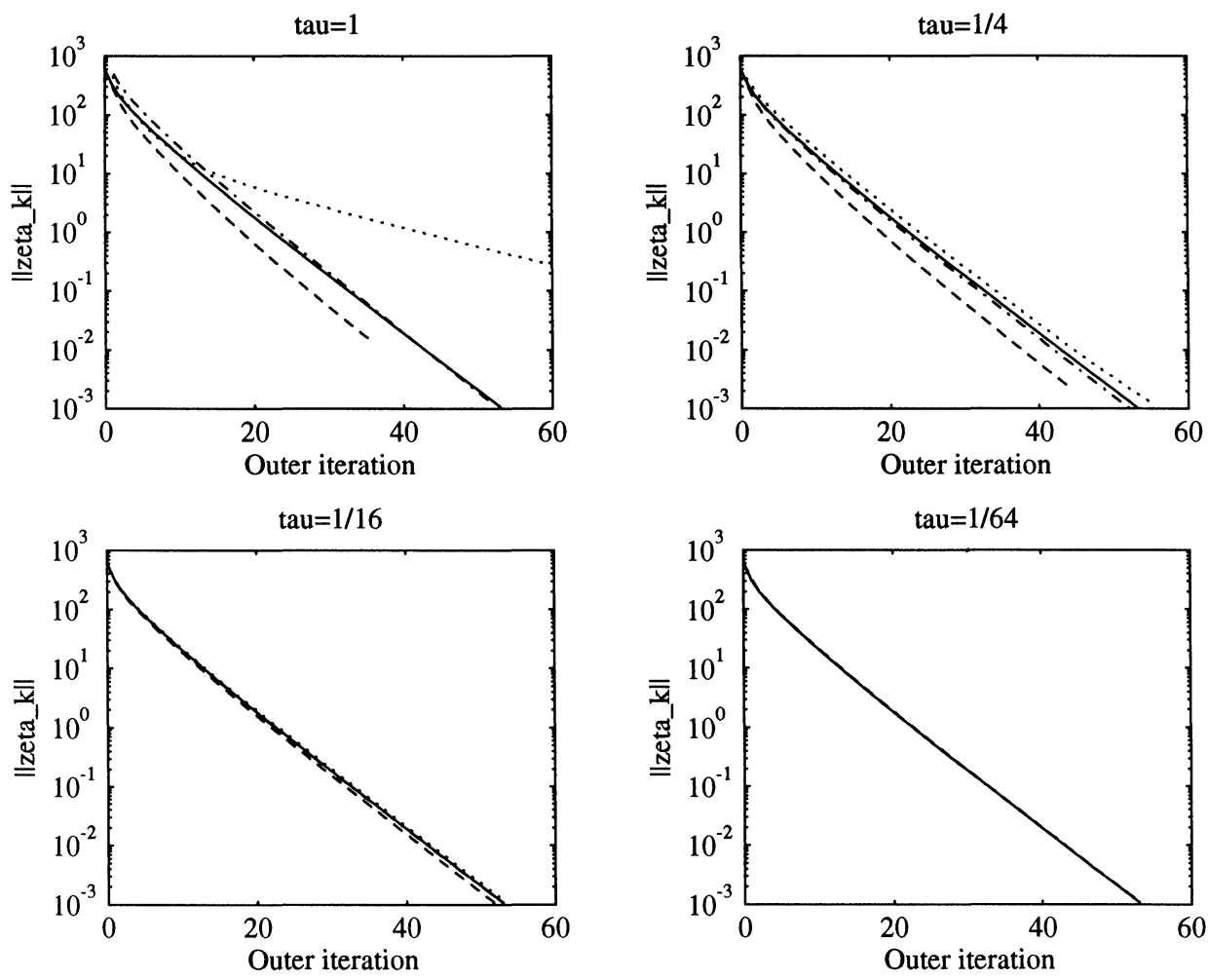

FIG. 1. Error norms $\zeta_{k}$ for the stabilized $P_{1}(h) P_{1}(h)$ discretization with $h=1 / 32$ and diagonal preconditioning, for four values of $\tau$. Direct solver: -, SOR: $\cdots \cdot$, MIC: - - -, Multigrid: - - - .

Table 3 compares estimates for the asymptotic convergence factors to the bounds for each of the discretizations, with $h=1 / 32$ and four values of $\tau$. We restrict our attention to the preconditioned versions of the problems; some results for the unpreconditioned algorithm are shown below. The estimates for the asymptotic convergence factors are

$$
\left(\zeta_{k} / \zeta_{k-10}\right)^{1 / 10}
$$

where $k$ is the index of the last iteration. (If this quantity is denoted by $\mu$, then $\log \mu$ is the slope of the line segment corresponding to the last 10 iterations of the computations, as in Figs. 1 and 2 below; these give a more accurate picture of the asymptotic behavior for finite $k$ than $\left(\zeta_{k} / \zeta_{0}\right)^{1 / k}$.) In the table, $\rho$ is the convergence factor, and $\rho_{e}$ is the observed factor, (33) for the exact Uzawa algorithm.

The bounds for all these tests, shown in the rightmost column of Table 3, indicate that the inexact algorithm is convergent even for large values of $\tau$; with one exception, they guarantee convergence for $\tau \leq 1 / 4$. Although the bounds are somewhat pessimistic, they are in good qualitative agreement with the asympotic factors for $\tau \leq 1 / 16{ }^{3}$

Next, we consider the behavior of the errors in the outer iterations. Figure 1 shows the error norms $\zeta_{k}$ for one choice of test problem, corresponding to the stabilized

\footnotetext{
${ }^{3}$ We attribute the fact that the bounds $\hat{\rho}$ are smaller than the observed convergence factors in some instances to the possibility that the asymptotic regime in $k$ has not been reached yet.
} 

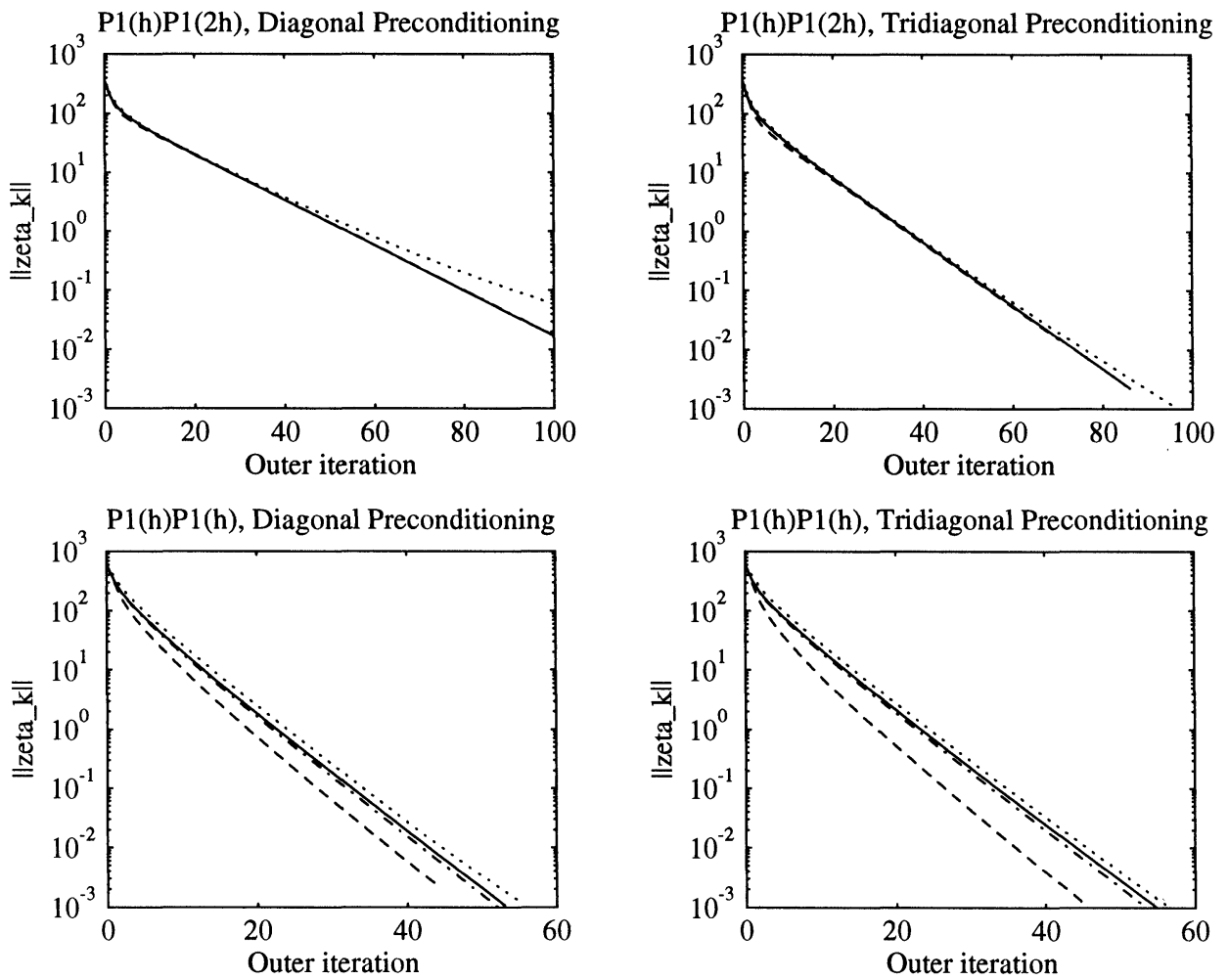

FIG. 2. Error norms $\zeta_{k}$ for four discrete problems with $h=1 / 32$ and $\tau=1 / 4$. Direct solver:

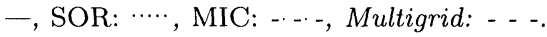

$P_{1}(h) P_{1}(h)$ discretization and diagonal preconditioning. Results are shown for each of the inner iteration strategies and the four values of $\tau$ used above, together with the error norms when a direct solve was used. The data indicate that the outer iteration counts are largely unaffected by the accuracy of the inner solve. These results are representative of the behavior for the other test problems. This is essentially indicated by the data of Table 3. In addition, Fig. 2 shows the error norms for four discrete problems, corresponding to two discretizations and two preconditioners for each, with $\tau=1 / 4$; except for the case of SOR with the $P_{1}(h) P_{1}(2 h)$ discretization, the errors for all the solution strategies display the same qualitative behavior.

A more important indicator of performance is the number of inner iterations required for convergence of the outer iteration. Results for this measure are shown in Table 4, for both the constant and linear pressure discretizations. Examination of any individual column of this data shows that in nearly all cases, the total number of inner iterations increases as the stopping criterion becomes more stringent. Numbers in parentheses correspond to cases where the outer iteration was not convergent within 500 steps.

Remark 5.1. Our primary objective in presenting the data shown thus far is to examine the performance of the inexact Uzawa algorithm. It is evident that the algorithm is effective for relatively modest requirements on the accuracy of the inner iteration.

Remark 5.2. It is not our aim to compare the effectiveness of the methods used 
TABLE 4

Number of inner iterations for convergence of the inexact Uzawa algorithm, for $h=1 / 32$.

\begin{tabular}{|c|c|c|c|}
\hline \multicolumn{4}{|c|}{$\begin{array}{c}P_{1}(h) P_{1}(2 h) \\
\text { Diagonal } \quad \text { preconditioner }\end{array}$} \\
\hline$\tau$ & SOR & MIC & MG \\
\hline 1 & $(6997)$ & 571 & 212 \\
\hline $1 / 4$ & 3902 & 795 & 415 \\
\hline $1 / 16$ & 3502 & 1057 & 585 \\
\hline $1 / 64$ & 3835 & 1290 & 836 \\
\hline \multicolumn{4}{|c|}{$\begin{array}{l}P_{1}(h) P_{1}(h) \\
\text { nal preconditioner }\end{array}$} \\
\hline$\tau$ & SOR & MIC & MG \\
\hline 1 & 2137 & 308 & 121 \\
\hline $1 / 4$ & 1317 & 462 & 217 \\
\hline $1 / 16$ & 1728 & 577 & 360 \\
\hline $1 / 64$ & 1900 & 662 & 472 \\
\hline
\end{tabular}

\begin{tabular}{c|ccc}
\multicolumn{4}{c}{$P_{1}(h) P_{1}(2 h)$} \\
\multicolumn{3}{c}{ Tridiagonal } \\
\multicolumn{1}{c|}{ SOR } & MIC & MG \\
\hline 1 & $(6962)$ & 391 & 209 \\
$1 / 4$ & 2145 & 586 & 335 \\
$1 / 16$ & 2677 & 845 & 488 \\
$1 / 64$ & 2979 & 955 & 668 \\
\multicolumn{4}{|c}{} \\
\multicolumn{4}{|c}{$P_{1}(h) P_{1}(h)$} \\
Tridiagonal preconditioner \\
\multicolumn{1}{c|}{ SOR } & MIC & MG \\
\hline 1 & 2065 & 314 & 153 \\
$1 / 4$ & 1405 & 489 & 222 \\
$1 / 16$ & 1830 & 599 & 374 \\
$1 / 64$ & 2059 & 690 & 490
\end{tabular}

TABLE 5

Effect of preconditioning on iteration counts for the inexact Uzawa algorithm with multigrid, for $h=1 / 32$.

\begin{tabular}{l|ccc|ccc}
\multicolumn{7}{c}{$P_{1}(h) P_{1}(2 h)$} \\
$\tau$ & None & Diagonal & Tridiagonal & None & Diagonal & Tridiagonal \\
\hline 1 & 431 & 97 & 70 & 481 & 212 & 209 \\
$1 / 4$ & 427 & 97 & 70 & 1045 & 415 & 335 \\
$1 / 16$ & 426 & 99 & 78 & 2144 & 585 & 488 \\
$1 / 64$ & $(500)$ & 105 & 84 & $(3996)$ & 836 & 668 \\
& \multicolumn{7}{c}{$\begin{array}{c}\text { Outer iterations } \\
\text { Inner iterations }\end{array}$}
\end{tabular}

\begin{tabular}{|c|c|c|c|c|c|c|}
\hline \multicolumn{7}{|c|}{$P_{1}(h) P_{1}(h)$} \\
\hline$\tau$ & None & Diagonal & Tridiagonal & None & Diagonal & Tridiagonal \\
\hline 1 & 79 & 36 & 39 & 277 & 121 & 153 \\
\hline $1 / 4$ & 100 & 44 & 45 & 497 & 217 & 222 \\
\hline $1 / 16$ & 129 & 52 & 54 & 899 & 360 & 374 \\
\hline $1 / 64$ & 135 & 53 & 55 & 1210 & 472 & 490 \\
\hline & & Outer ite & tions & & Inner iter & ions \\
\hline
\end{tabular}

for the inner solves. Their asymptotic properties as $h \rightarrow 0$ are well understood, with multigrid displaying the fastest convergence and SOR the slowest. With respect to their use in this context, however, several trends are worth pointing out. In particular, the convergence factors for SOR in Table 3 are closer to the upper bounds than those for either MIC or MG, and if the stopping criterion is not stringent, SOR leads to divergent outer iterations in some cases. In contrast, the convergence behavior for MG, and to a lesser extent for MIC, is better than expected for mild stopping criteria; indeed, in many cases with large $\tau$, convergence of the outer iteration is actually faster than when a direct solve is used. Moreover, with stringent stopping criteria ( $\operatorname{small} \tau$ ), all the inner iteration strategies often lead to smaller asymptotic convergence factors than the exact algorithm. See [33] for an analysis of these phenomena.

Finally, we consider the effect of preconditioning on performance. We restrict our attention to multigrid for the inner iteration. The iteration counts, for both the outer and inner iterations, are shown in Table 5 . These results are in agreement with those for convergence factors in $\S \S 3-4$ (see Table 1 ). For the $P_{1}(h) P_{1}(2 h)$ discretization, preconditioning by both the diagonal and tridiagonal parts of the mass matrix dramatically improves performance, and with only marginally extra cost per step, 
the tridiagonal part is the more effective choice. For the $P_{1}(h) P_{1}(h)$ discretization, the two preconditioners are actually about equally effective. We have observed that as $h$ decreases from $1 / 8$ to $1 / 64$, the convergence factors for the tridiagonal preconditioner grow less rapidly than for the diagonal one, and we expect the tridiagonal preconditioner to produce lower iteration counts as $h \rightarrow 0$. Once again, we also find that more stringent stopping criteria for the inner solve produces some degradation of performance, including an increase in the number of outer iterations.

Acknowledgments. We thank David Silvester, Andy Wathen, and Bruno Welfert for several very helpful discussions, and Jan Mandel for providing us with a copy of his multigrid code.

\section{REFERENCES}

[1] D. Arnold, F. Brezzi, And M. Fortin, A stable finite element for the Stokes equations, Calcolo, 21 (1984), pp. 337-344.

[2] K. Arrow, L. Hurwicz, And H. Uzawa, Studies in Nonlinear Programming, Stanford University Press, Stanford, CA, 1958.

[3] I. BABUŠKA, The finite element method with Lagrange multipliers, Numer. Math., 20 (1973), pp. 179-192.

[4] R. E. Bank, B. D. Welfert, and H. Yserentant, A class of iterative methods for solving saddle point problems, Numer. Math., 56 (1990), pp. 645-666.

[5] J. H. BRAmble AND J. E. PASCIAK, A preconditioning technique for indefinite systems resulting from mixed approximations of elliptic problems, Math. Comp., 50 (1988), pp. 1-17.

[6] A. Brandt and N. Dinar, Multigrid solutions to elliptic flow problems, in Numerical Methods for Partial Differential Equations, S. V. Parter, ed., Academic Press, New York, 1979, pp. 53-147.

[7] F. BREzzI, On the existence, uniqueness and approximation of saddle-point problems arising from Lagrange multipliers, RAIRO Anal. Numer., 8 (1974), pp. 129-151.

[8] F. Brezzi And M. Fortin, Mixed and Hybrid Finite Element Methods, Springer-Verlag, New York, 1991.

[9] F. Brezzi And J. PitKäranta, On the stabilisation of finite element approximations of the Stokes problem, in Efficient Solutions of Elliptic Systems, Notes on Numerical Fluid Mechanics, Vol 10, W. Hackbusch, ed., Vieweg, Braunschweig, 1984, pp. 11-19.

[10] J. Cahouet And J.-P. ChabARD, Some fast 3D finite element solvers for the generalized Stokes problem, Internat. J. Numer. Meth. Fluids, 8 (1988), pp. 869-895.

[11] R. S. Dembo, S. C. Eisenstat, and T. Steinaug, Inexact Newton methods, SIAM J. Numer. Anal., 19 (1982), pp. 400-408.

[12] T. Dupont, R. P. Kendall, and H. H. RachFord, JR., An approximate factorization procedure for solving self-adjoint elliptic difference equations, SIAM J. Numer. Anal., 5 (1968), pp. 559-573.

[13] M. Fortin And R. Glowinski, Augmented Lagrangian Methods: Applications to the Numerical Solution of Boundary-Value Problems, North-Holland, New York, 1983.

[14] I. FRIED, Bounds on the extremal eigenvalues of the finite element stiffness and mass matrices and their spectral condition number, J. Sound Vibration, 22 (1972), pp. 407-418.

[15] V. Girault And P. A. Raviart, Finite Element Approximation of the Navier-Stokes Equations, Springer-Verlag, New York, 1986.

[16] R. Glowinski, Numerical Methods for Nonlinear Variational Problems, Springer-Verlag, New York, 1984.

[17] G. H. Golub And M. L. Overton, The convergence of inexact Chebyshev and Richardson iterative methods for solving linear systems, Numer. Math., 53 (1988), pp. 571-593.

[18] G. H. Golub AND M. A. SAunders, Linear least squares and quadratic programming, in Integer and Nonlinear Programming, J. Abadie, ed., North-Holland, New York, 1970, pp. 229-256.

[19] M. Gunzburger, Finite Element Methods for Viscous Incompressible Flows, Academic Press, San Diego, 1989.

[20] I. GustafsSon, A class of first order factorizations, BIT, 18 (1978), pp. 142-156.

[21] Y. Maday, D. Meiron, A. T. Patera, And E. M. Ronquist, Analysis of iterative methods for the steady and unsteady Stokes problem: Application to spectral element discretizations, SIAM J. Sci. Comput., 14 (1993), pp. 310-337. 
[22] J. F. Maitre, F. Musy, AND P. Nigon, A fast solver for the Stokes equations using multigrid with a Uzawa smoother, in Advances in Multi-Grid Methods, Notes on Numerical Fluid Mechanics, Vol 11, D. Braess, W. Hackbusch, and U. Trottenberg, eds., Braunschweig, Vieweg, 1985, pp. 77-83.

[23] S. F. MCCORMICK, ED., Multigrid Methods, Society for Industrial and Applied Mathematics, Philadelphia, 1987.

[24] W. QUECK, The convergence factor of preconditioned algorithms of the Arrow-Hurwicz type, SIAM J. Numer. Anal., 26 (1989), pp. 1016-1030.

[25] T. RUSTEN AND R. Winther, A preconditioned iterative method for saddle point problems, SIAM J. Matrix Anal. Appl., 13 (1992), pp. 887-904.

[26] D. J. Silvester, Optimal low order finite element methods for incompressible flow, Comput. Meth. Appl. Mech. Engrg., 111 (1994), pp. 357-368.

[27] D. J. Silvester And A. Wathen, Fast Iterative Solution of Stabilized Stokes Systems Part II: Using General Block Preconditioners, SIAM J. Numer. Anal., 31(1994), pp. 1352-1367.

[28] R. S. VARGA, Matrix Iterative Analysis, Prentice-Hall, Englewood Cliffs, NJ, 1962.

[29] R. VERFüRTH, A combined conjugate gradient-multigrid algorithm for the numerical solution of the Stokes problem, IMA J. Numer. Anal., 4 (1984), pp. 441-455.

[30] A. Wathen And D. Silvester, Fast iterative solution of stabilized Stokes systems part I: Using simple diagonal preconditioners, SIAM J. Numer. Anal., 30 (1993), pp. 630-649.

[31] A. J. WATHEN, Realistic eigenvalue bounds for the Galerkin mass matrix, IMA J. Numer. Anal., 7 (1987), pp. 449-457.

[32] - Spectral bounds and preconditioning methods using element-by-element analysis for Galerkin finite element equations, in The Mathematics of Finite Elements and Applications VI, J. R. Whiteman, ed., Academic Press, London, 1987, pp. 157-168.

[33] B. D. Welfert, Convergence of Inexact Uzawa Algorithms for Saddle Point Problems, Tech. Rep., Mathematics Dept., Univ. of Arizona, 1993.

[34] G. WitTum, Multi-grid methods for the Stokes and Navier-Stokes equations, Numer. Math., 54 (1989), pp. 543-564.

[35] M. WRIGHT, Interior methods for constrained optimization, in Acta Numerica, Cambridge University Press, Cambridge, 1992, pp. 341-407.

[36] D. M. Young, Iterative Solution of Large Linear Systems, Academic Press, New York, 1970. 